\title{
BMJ Open Providing person-centred care for people with multiple chronic conditions: protocol for a qualitative study incorporating client and staff perspectives
}

\author{
Annette Peart (D) , Virginia Lewis, ${ }^{2}$ Christopher Barton, ${ }^{1}$ Ted Brown, ${ }^{3}$ Julie White, ${ }^{4}$ \\ Debra Gascard, ${ }^{4}$ Grant Russell ${ }^{1}$
}

To cite: Peart A, Lewis V, Barton C, et al. Providing person-centred care for people with multiple chronic conditions: protocol for a qualitative study incorporating client and staff perspectives. BMJ Open 2019;9:e030581. doi:10.1136/ bmjopen-2019-030581

- Prepublication history and additional material for this paper are available online. To view these files, please visit the journal online (http://dx.doi. org/10.1136bmjopen-2019030581).

Received 21 March 2019 Revised 19 August 2019 Accepted 20 August 2019

Check for updates

(C) Author(s) (or their employer(s)) 2019. Re-use permitted under CC BY-NC. No commercial re-use. See rights and permissions. Published by BMJ.

${ }^{1}$ Department of General Practice, Monash University, Notting Hill, Victoria, Australia

${ }^{2}$ Australian Institute for Primary Care and Aging, La Trobe University, Bundoora, Victoria, Australia

${ }^{3}$ Department of Occupational Therapy, Monash University, Frankston, Victoria, Australia ${ }^{4}$ Monash Health, Dandenong, Victoria, Australia

Correspondence to

Ms Annette Peart;

annette.peart@monash.edu

\section{ABSTRACT}

Introduction Chronic conditions are associated with over one-third of potentially avoidable hospitalisations. Integrated care programmes aim to help people with chronic conditions to self-manage their health, thus avoiding hospital admissions. While founded on principles of person-centred care, the experiences of people with multiple chronic conditions in integrated care programmes are not widely known. Our study will explore how personcentred care is incorporated into an integrated care programme for people with multiple chronic conditions. Methods and analysis This is a qualitative phenomenological study being conducted from March 2018 to June 2019, in a large metropolitan health service in Melbourne, Australia. Participants will be programme clients (and/or their carers) and staff working in the programme. We will interview staff about their experiences of the programme. Recruited staff will assist with recruitment of clients who recently completed an episode of care, to participate in a semistructured interview in their home. We will also analyse the medical records of interviewed clients, and observe outpatient clinics connected to the programme, based on the findings of the interviews. We will analyse all data using thematic analysis, with overarching themes representing staff and client perspectives of person-centred care.

Ethics and dissemination Ethical approval was granted by Monash Health (HREC/18/MonH/33) and Monash University (12260) Human Research Ethics Committees. Our study will provide a comprehensive exploration of person-centred care in an integrated care programme. It will add information to person-centred care literature on participants' perceptions of what works and why, including barriers and enablers to person-centred care in a complex environment. Findings of this study will be disseminated via publications, conferences and presentations to the health service participants.

\section{INTRODUCTION}

Chronic conditions are associated with over one-third of potentially avoidable hospitalisations in Australia and more than $60 \%$ of the total burden of disease. ${ }^{2}$ To address
Strengths and limitations of this study

- We designed this study to explore in-depth staff and clients' experiences of an integrated care programme, based on principles of person-centred care.

- A qualitative approach comprising interviews, document analysis and observation will ensure the credibility of the findings, and clear justification of the themes.

- The recruitment strategy may limit the transferability of our findings to similar settings; however, this will allow us to gain a rich description of the experiences of participants.

this, a range of programmes and services that focus on hospital avoidance aim to assist people with chronic conditions to manage their disease symptoms and overall health, by coordinating services across primary healthcare, specialist services and hospitals, thereby reducing hospitalisations.

Oneapproach,integrated care programmes, aim to provide coordinated, proactive, person-centred, multidisciplinary care by two or more collaborating care providers, in the same or different health or social care organisations. ${ }^{3}$ Integrated care is a broad term that is used interchangeably with coordinated or comprehensive care programmes, ${ }^{45}$ chronic disease management ${ }^{6}$ or hospital avoidance programmes, such as the Hospital Admission Risk Program (HARP) in Australia. ${ }^{7}$ Recent work demonstrates these programmes may reduce hospital admissions, emergency department (ED) presentations and length of stay. ${ }^{8-14}$

The Chronic Care Model ${ }^{15}$ is the framework most frequently cited in studies on integrated care for people with multiple chronic 
conditions, ${ }^{3}$ (or multimorbidity, defined in this paper as two or more chronic conditions in the same person). ${ }^{16}$ The model incorporates dimensions of patient-centred (or person-centred) care into how health service systems must be 'organised' for chronic illness care. ${ }^{17}$ The elements of the Chronic Care Model are designed to make person-centred, evidence-based care for people with chronic conditions easier to accomplish. ${ }^{18}$

A 2012 systematic review of characteristics and effectiveness of care programme for patients with multimorbidity found moderate evidence for a beneficial effect of these programmes on perceived quality of care, ${ }^{5}$ but inconclusive evidence for effectiveness overall. This review was repeated in 2015 by different authors, expecting to find stronger evidence. ${ }^{4}$ For patient outcomes, the updated review found insufficient evidence for a beneficial effect of comprehensive care on satisfaction with care and quality of life. The review authors recommended a focus on measuring outcomes that really matter to patients, such as personal goal attainment.

Person-centred care is a key dimension of high-quality healthcare. ${ }^{19}$ It has been characterised as a multidimensional concept, interpreted in different ways according to context. ${ }^{20-23} \mathrm{~A}$ narrative review and synthesis of key medical, nursing and policy texts identified three core themes of patient-centred care: patient participation and involvement, the relationship between the patient and health professional and the context where care is delivered. ${ }^{24}$ One of the core principles underlying integrated care programmes is person-centred care, achieved via collaborative, mutual and respectful partnerships between the provider and the person receiving care. $^{7}$

The Health Foundation has developed an enabling model of person-centred care, focusing on the person taking an active role in their health and health decisions. ${ }^{25}$ This model resonates with integrated care, and the Chronic Care Model, with a focus on chronic disease self-management and support. The enablement model describes four key principles of person-centred care:

1. Being person-centred means affording people dignity, respect and compassion.

2. Being person-centred means offering coordinated care, support or treatment.

3. Being person-centred means offering personalised care, support or treatment.

4. Being person-centred means being enabling.

Evidence of the efficacy of person-centred care as an intervention is limited due to problems in design and execution of studies, variations in terms, definitions and measures and different processes used in different settings. ${ }^{26-28} \mathrm{~A}$ recent systematic review of patient-centred interventions for people with chronic heart failure focused on patient involvement in developing care plans, sharing control and patients identifying their own care goals. ${ }^{26}$ In that review, patient-centred care was found to improve health-related quality of life, symptom burden, depression and patient activation; yet conclusions were limited by underpowered studies, and low to moderate strength of evidence. ${ }^{26}$

Research on interventions for people with multimorbidity has an overemphasis on effectiveness and an under-representation of qualitative evaluation. ${ }^{29} \mathrm{~A}$ better understanding of not only the health priorities of people with multimorbidity but their experiences of person-centred care is required. ${ }^{22}{ }^{2930}$ While the Chronic Care Model is widely used in integrated care programmes, there is a need for further research to understand the experiences, needs and preferences of people with multiple chronic conditions receiving integrated care, and their role in achieving positive outcomes in their care..$^{3-5} 14$ Some studies have turned to clinicians and researchers to describe how they define and deliver person-centred care. ${ }^{31-33}$ Their perceptions are valuable; however, the best way to understand how person-centred care is received is by asking patients themselves directly. ${ }^{345}$ The preferences people have about approaches to their care and what is most meaningful to them need to be understood in more depth and investigated further. ${ }^{36-38}$

This project aims to explore how an integrated care programme for people with multiple chronic conditions incorporates the principles of person-centred care. Our investigation will focus on (1) the clients' experience of care and (2) the individual and organisational routines supporting the delivery of care, through answering the following research questions:

1. What are the experiences of people with chronic conditions in planning and enacting their care plan?

2. What are the experiences of people with chronic conditions in using information provided by health professionals to make decisions about their care?

3. What characteristics of person-centred care matter most to people with chronic conditions?

4. How does the programme identify and respond to the needs of people with chronic conditions?

5. For health professionals, what are the barriers and enablers to providing person-centred care?

\section{METHODS AND ANALYSIS \\ Qualitative approach}

We want to understand person-centred care from the perspective of those experiencing it by asking them directly, ${ }^{34}$ therefore we will use a qualitative phenomenological $^{39}$ approach. In particular, we will take a hermeneutic phenomenological perspective, interpreting and describing human experience to understand the nature of that experience. ${ }^{40}$ This study will help us understand how people with multiple chronic conditions and complex needs experience an integrated care programme designed to reduce their hospitalisations and improve their ability to manage their health.

\section{Context}

The setting is a large metropolitan health service in Victoria, Australia, in an area of rapid population 
growth, with population groups who are disadvantaged in accessing services, due to cultural, linguistic or socioeconomic factors. The health service comprises three large research and teaching hospitals which serve over one-quarter of the city's population (around 1.9 million people), alongside several community health service locations. We selected the health service based on our existing relationships.

The health service provides integrated care through Hospital Admission Risk Program (HARP) services to manage people with chronic disease, aged and/ or complex needs who frequently use hospitals or are at risk of hospitalisation. One programme under the HARP umbrella of services is called the Complex Care Program. The key objectives of this programme are to improve patient outcomes, provide integrated, seamless care within and across hospital and community services, reduce avoidable hospital admissions and ED presentations and ensure equitable access to healthcare.

The Complex Care Program, guided by the Chronic Care Model, ${ }^{41}{ }^{42}$ provides integrated, community-based, multidisciplinary care and support across the secondary and tertiary levels of the health service, and into primary care and other community health and social care providers. The target population is people with chronic conditions and complex needs who frequently use hospitals or are at risk of hospitalisation. The programme comprises intensive care coordination provided by clinical nurse consultants, comprehensive assessment and care planning, specialist medical management and a self-management approach. ${ }^{7}$ Complex Care clients may be enrolled in the programme from 6 to 12 months, in one of the following streams: chronic heart failure, chronic respiratory disease and complex psychosocial conditions. In addition, specialist clinics support the management of clients with complex medical needs. A physician, nurse and pharmacist assess people attending the clinics to determine areas of their health requiring further investigation, streamlining of medication or reorganisation of services.

\section{Sampling strategy}

There are two main groups of participants in this study: Complex Care staff and Complex Care clients, which may include clients' informal carers.

\section{Staff participants}

The Complex Care Program employs 37 staff consisting of a manager, 3 team leaders, 20 care coordinators, 5 physicians, 7 allied health professionals (eg, physiotherapy, psychology, pharmacy, dietetics) and 1 chronic disease nurse practitioner. Care coordinators are predominantly clinical nurse consultants, expert registered nurses with skills and expertise in specific areas of practice. Programme staff provide intensive care coordination and home-based or centre-based services determined by the client's need. Staff have specific skill sets and experience in care coordination, specialist medical care, self-management support and the management of complex psychosocial issues. Exploration of the experience of providing person-centred care, and programme staff perceptions of this experience, is congruent with a hermeneutic phenomenological approach. ${ }^{43}$

Programme staff will participate in this study to provide their perspectives of person-centred care, including barriers and enablers to providing care. They will also assist with recruitment of client participants (see Recruitment procedure).

\section{Client participants}

Programme clients have multiple chronic conditions: unstable or complicated chronic respiratory disease, chronic heart failure, diabetes or other chronic conditions and/or complex care requirements. Most live independently in the community, although some older clients living in long-term aged care can also access a programme. Clients have had at least one unnecessary or avoidable hospital presentation or admission in the 12 months preceding recruitment, or are at imminent risk of hospitalisation and other community services cannot meet their needs. They also require integrated care and moderate to intensive care coordination. Over 1000 people access a Complex Care Program in this setting each year. Most clients are adults yet the service also works with children and youth. Exclusion criteria for this study are addressed in the Recruitment procedure section.

\section{Recruitment procedure}

We will purposively recruit staff based on their experience in the Complex Care Program and working with people with chronic conditions, role in the programme, and ability to reflect on their professional practice and explain how and why they work in a particular way. The Complex Care Manager will select an initial sample of up to 12 staff based on these criteria. We expect many staff will be care coordinators as they are most likely to have longitudinal relationships with their clients and extensive experience in the programme. We will exclude the recruitment of staff who are new to the programme (eg, commenced $<3$ months ago). During the data collection phase, we will ask the recruited staff of any additional staff members to recruit, adding 'snowball sampling' as an additional strategy for this study. The final size and composition of the sample will be determined during data collection and in the early stages of data analysis based on the richness of the information. ${ }^{39}$

We will recruit clients via their care coordinators. After care coordinators are interviewed, we will discuss with them the selection of up to five 'information-rich' clients recently discharged from the programme: clients from whom we can learn extensively about their experiences of service provision and person-centred care. ${ }^{43}{ }^{44}$ We will exclude clients enrolled in an existing research project with the health service, or if there are safety concerns for the researchers when interviewing the client. If a client has an impairment that precludes them from consenting and 
participating, as reported by the care coordinators based on their knowledge of the client, or any formal testing that may have occurred during the client's programme (eg, a Mini-Mental State Examination ${ }^{45}$ ), they may still be able to participate. In this scenario, we will ask the client's carer to assist with explaining the study to the client, and/ or provide their own consent to be interviewed about their experiences of the programme as a carer.

We anticipate the initial sample size of staff as up to 12 (mostly made up of care coordinators, and other clinicians working on the programme), and clients (five per care coordinator) as up to 30 . The size of each participant (staff and client) sample will depend on the information richness of the data and the variety of participants interviewed. Sampling is aimed at insight into person-centred care, not generalisation. ${ }^{39}$ Our initial estimations of participant numbers may provide enough in-depth data to answer the relevant research questions. We will determine the final sample size during the study based on a model of information power. ${ }^{46}$ This model indicates that the more information the sample holds relevant for the research questions, the lower number of participants needed.

Interview Sampling and Recruitment Process outlines the sampling and recruitment process for the interviews (online supplementary file 1). The study is being conducted from March 2018 to June 2019. We will seek written informed consent from all participants in the study and ensure confidentiality through the use of pseudonyms for individual participants.

\section{Data collection}

Consistent with the qualitative approach, data will be collected using three methods: semistructured interviews, observation and analysis of medical records. ${ }^{47} \mathrm{We}$ will collect data primarily in relation to clients' and staff descriptions of their experiences. We will use key principles of person-centred care ${ }^{25}$ to frame data collection. Table 1 summarises the data collection methods related to each research question.
We will start data collection with semistructured interviews with staff and client participants, to elicit their descriptions of their experiences of the programme. Staff and clients will each participate in one semistructured interview of up to 1 hour with the first author.

If a client has a carer, we will invite the carer to participate in the interview and obtain their consent.

We will observe the routines of the multidisciplinary clinics that the programme clients attend to obtain contextual and environmental information alongside verbal descriptions of their experiences obtained during interviews. Observation will assist us to understand and capture the context within which staff and clients interact, providing a holistic perspective. ${ }^{39}$ We will observe formal and informal interactions including clinical routines and activities in non-clinical areas. This will help us understand the setting and how it operates, and how staff undertake their usual routines and interact with clients, using the lens of the key principles of person-centred care. ${ }^{21}$

On completion of client interviews, with the client's consent, we will review the medical record relating to the time they were on the programme. Client records in the programmes are a rich source of data to supplement observations and interviews; they may reveal thoughts, decisions and conversations that might be unknown through observation or interviews. ${ }^{39}$ This will allow us to obtain data on the components of their programme of care as well as note documentation of services provided consistent with key principles of person-centred care. ${ }^{21}$ We will record text referring to person-centred care in areas such as assessment, care planning, decision-making or provision of information or education.

\section{Data collection instruments and technologies}

We will use a semistructured interview guide based on the research questions and the key principles of person-centred care. ${ }^{2139}$ Separate interview guides were developed for staff and client participants as outlined in online supplementary file 2. After the first three interviews for staff and clients, we will review the transcript within the research

Table 1 Research questions and data collection summary

\begin{tabular}{|c|c|c|c|c|}
\hline Research questions & $\begin{array}{l}\text { Staff } \\
\text { interviews }\end{array}$ & $\begin{array}{l}\text { Client } \\
\text { interviews }\end{array}$ & Observation & $\begin{array}{l}\text { Document } \\
\text { analysis }\end{array}$ \\
\hline $\begin{array}{l}\text { What are the experiences of people with chronic } \\
\text { conditions in planning and enacting their care plan? }\end{array}$ & & $\checkmark$ & $\checkmark$ & \\
\hline $\begin{array}{l}\text { What are the experiences of people with chronic } \\
\text { conditions in using the information provided by health } \\
\text { professionals to make decisions about their care? }\end{array}$ & & $\checkmark$ & $\checkmark$ & \\
\hline $\begin{array}{l}\text { What characteristics of patient-centred care matter most } \\
\text { to people with chronic conditions? }\end{array}$ & $\checkmark$ & $\checkmark$ & & $\checkmark$ \\
\hline $\begin{array}{l}\text { For health professionals, what are the barriers and } \\
\text { enablers to providing patient-centred care? }\end{array}$ & $\checkmark$ & $\checkmark$ & $\checkmark$ & $\checkmark$ \\
\hline
\end{tabular}


team, reflect on the interview processes and adapt the questions within the interview guide as required. We will progressively modify the guides with the iterative process of data collection and analysis. We will conduct interviews in person or via telephone and record interviews using a digital audio recorder. The interviews will be transcribed verbatim. Field notes will be kept and completed by hand at the end of each interview in the form of a contact summary sheet to capture impressions and reflections on the interview. ${ }^{48}$ If a client's first language is not English, we will use a qualified interpreter provided by the health service to attend the interview.

We will develop a tool to collect relevant data from the medical records and observations based on ongoing analysis of the staff and client interview data. This will enable us to compare and contrast data collected from the interviews, medical records and observation. Throughout data collection, the first author will make reflective notes via memos to note key points, observations, decisions or thoughts as they arise.

\section{Data processing}

Following each interview, we will review each transcript along with the interview recording to check for accuracy, remove identifying information and anonymise data. We will enter field notes and excerpts from documents into word processing software and check for accuracy. Participants will be offered the opportunity to review the interview transcripts or a summary of the interview, at the end of each interview. This will allow participants to check the accuracy of the transcription and/or elaborate on their responses. All data will be organised according to type and date collected, then entered into NVivo $12^{49}$ for data management during the analysis. We will convert all hard copy data to digital format and store on a secure, password-protected server.

\section{Data analysis}

Data analysis will occur concurrently with data collection and the writing up of findings. ${ }^{50}$ We have chosen to use Braun and Clarke's approach to thematic analysis for our study. ${ }^{51}$ Braun and Clarke describe thematic analysis as a method to identify, analyse and report patterns (themes) within data. Alongside this process we will also be guided by Ricoeur's theory of interpretation, with a focus on explaining and then interpreting the text (interview transcripts, field notes and medical record excerpts) to further understand the experiences of participants. ${ }^{40} 52$

Braun and Clarke's approach involves six phases. Table 2 displays the six phases alongside components of our study aligned with each phase.

The first author will code staff interviews and client interviews as two large groups, and use other members of the research team to assist with confirming codes. As the data analysis is an iterative process, depending on the findings from the two groups, individuals within the groups may be compared. Analysis of data gathered from observations and documents will occur after the

Table 2 Data analysis process

Thematic analysis phase ${ }^{51}$
1. Familiarisation with the data: transcribing, reading and re-
reading, noting initial ideas

2. Generating initial codes across data set, collating data for each code

\section{Searching for themes: collating codes into potential} themes and gathering all data relevant to each theme

4. Reviewing themes: checking themes work on extracts and the entire data set, generating thematic 'map'

5. Defining and naming themes: refinement through ongoing analysis, generating clear definitions and names for themes

6. Producing the report: selecting compelling extracts, final analysis, relating back to research questions, producing a report

\section{Study components}

- Re-listen to the audio recording; initial note taking to record analytical notes.

- Reading and re-reading of the transcripts.

- Reading transcripts systematically, generating codes alongside important points in the text.

Sorting codes into themes and collating all data extracts from the text. ${ }^{49}$

Using NVivo V.12 to assist with collating data extracts.

- Re-reading data extracts to look for patterns.

- Comparing codes generated from the first five transcripts with the research team and agreeing on a set of codes for subsequent transcripts.

- Coding all transcripts looking for additional data that may have been missed.

- Generating a working analytical 'map' using the developed codes and themes.

- Naming and defining the themes and sharing with the research team for review.

- Refining themes and cross-referencing with principles of person-centred care.

Writing a detailed analysis of each theme.

Telling the story of the data: interpreting the meaning of descriptions of participants' experiences.

- Embedding examples in the text to make an argument in relation to the research questions. 
Table 3 Techniques to enhance trustworthiness

\begin{tabular}{|c|c|}
\hline Lincoln and Guba's criteria ${ }^{53}$ & Techniques used in this study to enhance trustworthiness \\
\hline Credibility & $\begin{array}{l}\text { Member checking }{ }^{53} \text { : asking participants if they wish to review interview transcripts and } \\
\text { preliminary findings. } \\
\text { Triangulation of data sources: data gathering via staff and client interviews, observation } \\
\text { and document analysis, to build a coherent justification for themes. }{ }^{50} 555\end{array}$ \\
\hline Transferability & $\begin{array}{l}\text { Purposeful sampling of staff and client participants to maximise the range of data } \\
\text { collected. } \\
\text { 'Thick' descriptive data collected via interviews to provide enough information to be } \\
\text { able to compare these findings to other programmes in similar contexts. }{ }^{53} \text { Context is an } \\
\text { important consideration in the exploration of person-centred care. }{ }^{21}\end{array}$ \\
\hline Dependability & $\begin{array}{l}\text { 'Audit trail' to document and adhere to data collection and analysis processes and } \\
\text { interpretation. }{ }^{56} \\
\text { 'Dependability' audit undertaken by the first author's supervisors (CB, GR, VL) to check } \\
\text { the acceptability of the audit trail. }{ }^{53}\end{array}$ \\
\hline
\end{tabular}

interviews are analysed. Codes derived from the interviews will be applied to observation and document data.

\section{Techniques to enhance trustworthiness}

Lincoln and Guba $^{53}$ consider trustworthiness important for evaluating qualitative research. Trustworthiness involves establishing credibility, transferability, dependability and confirmability of the findings. We will use a variety of techniques to enhance trustworthiness in this study (table 3).

The first author (AP) is a female, registered occupational therapist with a Masters of Health Science degree and completing a Doctor of Philosophy at Monash University. She has over 20 years' postgraduate experience in rehabilitation. DG and JW are employees of the health service, with significant experience in quality improvement, particularly in nursing and community care. They will assist with recruitment of participants. GR is a general practitioner; GR, CB and VL are primary care researchers and AP's supervisors for this study. AP was introduced to DG and JW via an existing connection between GR and the health service. TB is a registered occupational therapist and associate professor of occupational therapy, who provided supervision to AP in the initial months of this project.

The Standards for Reporting Qualitative Research ${ }^{54}$ will be used to report the research to improve its transparency, usability and reliability.

\section{ETHICS AND DISSEMINATION}

All participants will receive complete written and verbal information about the research prior to giving full, non-coercive consent in accordance with the ethical guidelines. Participants are able to withdraw from participation at any time, without impacting on either their employment at the health services, or any future services they may be eligible for or receive. Procedures will be followed to minimise any potential harm or distress to participants, including the provision of contact details for further assistance (available at no cost to the participant) if required. Participant privacy and confidentiality will be respected by the removal of any identifying information from data, assigning pseudonyms and storing all data safely on password-protected systems or in locked cabinets at the university. Data will be accessible only to AP, CB, GR and VL. All data will be destroyed after 7 years in accordance with the agreed ethical standards.

We will disseminate the results of this study via presentations at relevant local, national and international conferences, peer-reviewed journals and through social media including Twitter accounts of AP, CB and GR, as well as those of the Department of General Practice, Monash University, and the Innovative Models Promoting Access-to-Care Transformation (IMPACT) Centre of Research Excellence. Only anonymised, non-identifiable characteristics and quotations will be used in any arising publications/reports.

\section{DISCUSSION}

Our study aims to understand how person-centred care is incorporated in an integrated care programme designed to assist people with multiple chronic conditions to manage their health using community-based health and social care supports, as well as to reduce their use of acute hospitals for treatment. It will focus on staff and client descriptions of their experiences in the programme, identify the characteristics of care important to them and the barriers and enablers to providing person-centred care. This involves interviewing staff, a sample of their recently discharged clients, observing staff and clients in specialist 
clinics and collecting and analysing key client and organisational documents.

\section{Limitations}

While following a client's programme from start to end would provide rich data, they may be reluctant to accept care during the initial phases of a programme or participate in research. We will interview clients who have finished a programme so they can reflect on their experiences of recent events.

We will purposefully select staff who we assume can provide rich data on their experiences of person-centred care. These staff must be able to clearly reflect and articulate their professional practice. However, not all staff may wish to go over their ways of working with the clients or discuss their practice preferences. Only interviewing those with an interest in the study will provide rich and comprehensive data of their experiences. Similarly for clients, by interviewing only interested participants to tell their story of the programme, regardless of the programme's impact on their health and well-being, we hope will provide rich descriptions of their experiences.

Beyond the challenges, our study will provide a comprehensive exploration of person-centred care in a programme that works to help reduce unnecessary hospitalisations. It will add information to person-centred care literature on participants' perceptions of what works and why, including the barriers and enablers to practicing person-centred care in a complex environment. The qualitative approach will allow participants to contribute to shaping service delivery in such programmes and has the potential to highlight future research opportunities incorporating the voices of clients and staff.

\section{Twitter Annette Peart @AnnettePeart}

Contributors AP, DG, GR, JW, TB and VL conceived of the study design. AP prepared the first draft of the manuscript. CB, DG, GR, JW, TB and VL all provided edits and approved the final version.

Funding This work was supported by the Innovative Models Promoting Accessto-Care Transformation (IMPACT) Centre of Research Excellence (CRE). The IMPACT CRE comprises the Canadian Institutes of Health Research Signature Initiative in Community-Based Primary Health Care, Fonds de recherche du Québec-Santé and the Australian Primary Health Care Research Institute, which is supported by a grant from the Australian Government Department of Health, under the Primary Health Care Research, Evaluation, and Development Strategy.

Competing interests None declared.

Patient consent for publication Not required.

Ethics approval Ethical approval for this study was granted by Monash Health (HREC/18/MonH/33) and Monash University (12260) Human Research Ethics Committees, in February 2018. Data collection and analysis will be conducted in accordance with National Health and Medical Research Council ethical guidelines and those of the approved Ethics Committees.

Provenance and peer review Not commissioned; externally peer reviewed.

Open access This is an open access article distributed in accordance with the Creative Commons Attribution Non Commercial (CC BY-NC 4.0) license, which permits others to distribute, remix, adapt, build upon this work non-commercially, and license their derivative works on different terms, provided the original work is properly cited, appropriate credit is given, any changes made indicated, and the use is non-commercial. See: http://creativecommons.org/licenses/by-nc/4.0/.
ORCID iD

Annette Peart http://orcid.org/0000-0002-0536-276X

\section{REFERENCES}

1 Australian Institute of Health and Welfare (AIHW). Admitted patient care 2013-14: Australian hospital statistics. Canberra, ACT: AlHW, 2015.

2 Australian Institute of Health and Welfare (AlHW). Australian burden of disease study: impact and cause of illness and death in Australia 2011. Australian burden of disease study series no 3. Canberra, ACT: AlHW, 2016.

3 Struckmann V, Leijten FRM, van Ginneken E, et al. Relevant models and elements of integrated care for multi-morbidity: results of a scoping review. Health Policy 2018;122:23-35.

4 Hopman P, de Bruin SR, Forjaz MJ, et al. Effectiveness of comprehensive care programs for patients with multiple chronic conditions or frailty: a systematic literature review. Health Policy 2016;120:818-32.

5 de Bruin SR, Versnel N, Lemmens LC, et al. Comprehensive care programs for patients with multiple chronic conditions: a systematic literature review. Health Policy 2012;107:108-45.

6 Reynolds R, Dennis S, Hasan I, et al. A systematic review of chronic disease management interventions in primary care. BMC Fam Pract 2018;191:11.

7 Department of Human Services. Health independence programs guidelines. Melbourne, Victoria: Victorian Government Department of Human Services, 2008.

8 Lawn S, Zabeen S, Smith D, et al. Managing chronic conditions care across primary care and hospital systems: lessons from an Australian hospital avoidance risk program using the Flinders chronic condition management program. Aust. Health Review 2018;42:542-9.

9 Bird S, Noronha M, Sinnott H. An integrated care facilitation model improves quality of life and reduces use of hospital resources by patients with chronic obstructive pulmonary disease and chronic heart failure. Aust J Prim Health 2010;16:326-33.

10 Howard R, Sanders R, Lydall-Smith SM. The implementation of Restoring Health - a chronic disease model of care to decrease acute health care utilization. Chron Respir Dis 2008;5:133-41.

11 Bird SR, Kurowski W, Dickman GK, et al. Integrated care facilitation for older patients with complex health care needs reduces hospital demand. Aust. Health Review 2007;31:451-61.

12 Roberts RM, Dalton KL, Evans JV, et al. A service model of short-term case management for elderly people at risk of hospital admission. Aust. Health Review 2007;31:173-83.

13 Joubert L, Power R. Using data-mining to explore the outcomes of an integrated care program: looking for meaning behind key outcome indicators. J Soc Work Res Eval 2005;6:185-94.

14 Damery S, Flanagan S, Combes G. Does integrated care reduce hospital activity for patients with chronic diseases? An umbrella review of systematic reviews. BMJ Open 2016;6:e011952.

15 Wagner EH, Austin BT, Davis C, et al. Improving chronic illness care: translating evidence into action. Health Aff 2001;20:64-78.

16 Smith SM, Wallace E, O'Dowd T, et al. Interventions for improving outcomes in patients with multimorbidity in primary care and community settings. Cochrane Database Syst Rev 2016;9.

17 Wagner EH, Bennett SM, Austin BT, et al. Finding common ground: Patient-Centeredness and evidence-based chronic illness care. The Journal of Alternative and Complementary Medicine 2005;11(supplement 1):s-7-s-15.

18 Coleman K, Austin BT, Brach C, et al. Evidence On The Chronic Care Model. In: The new millennium. . Health Aff (Millwood), 2009: 281. 75-85.

19 Australian Commission on Safety and Quality in Healthcare (ACSQH).. Patient-Centred care: improving quality and safety through partnerships with patients and consumers. Sydney: ACSQH, 2011.

20 Leplege A, Gzil F, Cammelli M, et al. Person-centredness: conceptual and historical perspectives. Disabil Rehabil 2007;29:1555-65.

21 Harding E, Wait S, Scrutton J. The state of play in person-centred care. The Health Policy Partnership Ltd, 2015.

22 Waters RA, Buchanan A. An exploration of person-centred concepts in human services: a thematic analysis of the literature. Health Policy 2017;121:1031-9.

23 Marshall A, Kitson A, Zeitz K. Patients' views of patient-centred care: a phenomenological case study in one surgical unit. $J$ Adv Nurs 2012;68:2664-73.

24 Kitson A, Marshall A, Bassett K, et al. What are the core elements of patient-centred care? A narrative review and synthesis of the literature from health policy, medicine and nursing. $J$ Adv Nurs 2013;69:4-15. 
25 Collins A. Measuring what really matters: towards a coherent measurement system to support person-centred care 2014

26 Olsson L-E, Jakobsson Ung E, Swedberg K, et al. Efficacy of personcentred care as an intervention in controlled trials - a systematic review. J Clin Nurs 2013;22:456-65.

27 Rathert C, Wyrwich MD, Boren SA. Patient-centered care and outcomes. Med Care Res Rev 2013;70:351-79.

28 McMillan SS, Kendall E, Sav A, et al. Patient-Centered approaches to health care: a systematic review of randomized controlled trials. Med Care Res Rev 2013;70:567-96.

29 Kastner M, Hayden L, Wong G, et al. Underlying mechanisms of complex interventions addressing the care of older adults with multimorbidity: a realist review. BMJ Open 2019;9:e025009.

30 Park M, Giap T-T-T, Lee M, et al. Patient- and family-centered care interventions for improving the quality of health care: a review of systematic reviews. Int J Nurs Stud 2018;87:69-83.

31 Fix GM, VanDeusen Lukas C, Bolton RE, et al. Patient-centred care is a way of doing things: how healthcare employees conceptualize patient-centred care. Health Expect 2018;21:300-7.

32 Moore L, Britten N, Lydahl D, et al. Barriers and facilitators to the implementation of person-centred care in different healthcare contexts. Scand J Caring Sci 2017;31:662-73.

33 Britten N, Moore L, Lydahl D, et al. Elaboration of the Gothenburg model of person-centred care. Health Expect 2017;20:407-18.

34 Stewart M. Towards a global definition of patient centred care. BMJ 2001;322:444-5.

35 Edvardsson D, Watt E, Pearce F. Patient experiences of caring and person-centredness are associated with perceived nursing care quality. J Adv Nurs 2017;73:217-27.

36 Lines LM, Lepore M, Wiener JM. Patient-centered, person-centered, and person-directed care: they are not the same. Med Care 2015;53:561-3.

37 Hudon C, Fortin M, Haggerty JL, et al. Measuring patients' perceptions of patient-centered care: a systematic review of tools for family medicine. The Annals of Family Medicine 2011;9:155-64.

38 Kane PM, Murtagh FEM, Ryan K, et al. The gap between policy and practice: a systematic review of patient-centred care interventions in chronic heart failure. Heart Fail Rev 2015;20:673-87.

39 Patton MQ. Qualitative research and evaluation methods: Integrating theory and practice. In. 4th ed. Los Angeles, CA: SAGE Publications, 2015.

40 Tan H, Wilson A, Olver I. Ricoeur's theory of interpretation: an instrument for data interpretation in Hermeneutic phenomenology. International Journal of Qualitative Methods 2009;84:1-15.

41 Wagner EH, Austin BT, Von Korff M, Korff MV. Organizing care for patients with chronic illness. Milbank Q 1996;74:511-44.
42 DoH S. Revised Chronic Disease Management Program Guidelines for Primary Care Partnerships and Primary Health Care Services. In: Department of human services PCB. Melbourne: Victorian Government Department of Human Services, 2008.

43 Laverty SM, Phenomenology H. Hermeneutic phenomenology and phenomenology: a comparison of historical and methodological considerations. Int J Qual Methods 2003;2:21-35.

44 Liamputtong P. Research methods in health: foundations for evidence-based practice. 3rd ed. South Melbourne, Victoria: Oxford University Press, 2017.

45 Folstein MF, Folstein SE, McHugh PR. "Mini-mental state". A practical method for grading the cognitive state of patients for the clinician. J Psychiatr Res 1975;12:189-98.

46 Malterud K, Siersma VD, Guassora AD. Sample size in qualitative interview studies: guided by information power. Qual Health Res 2015;26:1753-60.

47 Sloan A, Bowe B. Phenomenology and hermeneutic phenomenology: the philosophy, the methodologies, and using hermeneutic phenomenology to investigate lecturers' experiences of curriculum design. Qual Quant 2014;48:1291-303.

48 Miles M, Huberman AM. Qualitative data analysis: an expanded sourcebook. Thousand Oaks, CA: Sage Publications, 1994.

49 QSR International Pty Ltd. NVivo qualitative data analysis software, 2018.

50 Creswell JW. Research design: qualitative, quantitative, and mixed methods approaches. 4th ed. Thousand Oaks, CA: SAGE Publications, Inc, 2014.

51 Braun V, Clarke V. Using thematic analysis in psychology. Qual Res Psychol 2006;3:77-101.

52 Ricoeur P. The model of the text: meaningful action considered as a text. In: Thompson JB, Ricoeur P, eds. Hermeneutics and the human sciences: essays on language, action and interpretation. Cambridge Philosophy Classics: Cambridge: Cambridge University Press, 2016: 159-83.

53 Lincoln YS, Guba EG, Pilotta JJ. Naturalistic inquiry. Beverly Hills, CA: Sage Publications, 1985: 9. 438-9.

54 O'Brien BC, Harris IB, Beckman TJ, et al. Standards for reporting qualitative research: a synthesis of recommendations. Acad Med 2014;89.

55 Mays N, Pope C. Qualitative research: rigour and qualitative research. BMJ 1995;311:109-12.

56 Guba EG. Criteria for assessing the trustworthiness of naturalistic inquiries. Educational Communication and Technology 1981;29:75-91.

57 Birks M, Chapman Y, Francis K. Memoing in qualitative research: probing data and processes. J Res Nurs 2008;13:68-75. 